\title{
The Enskog theory for transport coefficients of simple fluids with continuous potentials
}

\author{
Kunimasa Miyazaki ${ }^{a}$ \\ IRI, Delft University of Technology, 2629 JB Delft, The Netherlands \\ Goundla Srinivas and Biman Bagchib) \\ Solid State and Structural Chemistry Unit, Indian Institute of Science, Bangalore 560 012, India
}

(Received 25 September 2000; accepted 17 January 2001)

\begin{abstract}
The Enskog theory for the self-diffusion coefficient for fluids with continuous potentials, such as the Lennard-Jones, is developed. Starting from the Green-Kubo formula (rather than the conventional kinetic equation) and introducing the similar assumptions upon which the Boltzmann equation is based, we derived a general expression for the memory kernel and the self-diffusion coefficient. The numerical analysis is implemented for the Lennard-Jones fluid. The time-dependent memory kernel is calculated and compared with the latest molecular dynamics simulations. Excellent agreement is obtained at the low density. The self-diffusion coefficient is evaluated for various temperatures and densities. The ratio of the Enskog self-diffusion coefficient to the simulation value is plotted against density. Significant difference of this density dependence from that for the hard-sphere fluid is observed. In particular, the well-known maximum observed (in the diffusion versus density plot) for the hard sphere fluid is found to be completely absent in the Lennard-Jones fluid. Our results reduce to the conventional Chapman-Enskog expression in the low density limit and can be applicable to the systems with singular potentials such as the hard sphere. (c) 2001 American Institute of Physics. [DOI: $10.1063 / 1.1355978]$
\end{abstract}

\section{INTRODUCTION}

Since its introduction in 1922, the Enskog theory for the hard-sphere fluid is known to be a very good approximation to describe the transport phenomena in the dense fluids (see Ref. 1 and references therein for details). Although it is a simple and empirical generalization of the Boltzmann equation, its usefulness is beyond dispute. For example, the shear viscosity calculated from the Chapman-Enskog theory which is a scheme to calculate the transport coefficients from the Boltzmann equation, is 40 times smaller in the dense liquid region than the simulation value, whereas the value evaluated using the Enskog theory is only 2 times as small at most. ${ }^{2,3}$ The Enskog approximation is not only practically useful in the dense gas region, but it also plays an important role in theoretical attempts to bridge the gap between the theory and the simulation values in the liquid region. As the density increases, each collision of atoms is not independent anymore and the so-called ring collisions become important. The most well-known scheme to take these dynamically correlated collisions into account is the mode-coupling theory (MCT).${ }^{4-6}$ Most of the input functions necessary to the MCT scheme such as the density correlation function are usually evaluated using the Enskog theory for the hard-sphere fluid.

Actually, the following assumptions upon which the Enskog theory is based are quite simple, though none of them is completely understood. ${ }^{1}$ (1) Each collision is completely independent and all multiple collisions except for the binary

\footnotetext{
${ }^{a)}$ Electronic mail: K.Miyazaki@iri.tudelft.nl

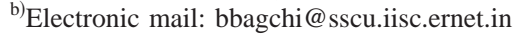

collision are neglected. (2) The frequency of the binary collision is increased by an amount which is proportional to the probability for one atom to find its neighbors. (3) Each collision process should be instantaneous.

The Enskog theory, however, is fully developed at present only for the hard-sphere fluid and in the present form cannot be applied to more realistic continuous potentials. It is not possible to generalize Enskog's argument to treat a continuous potential in a straightforward manner because the collisions are not instantaneous for the continuous potentials and, therefore assumption (3) is not valid. However, considering the usefulness of the Enskog theory for the hard-sphere fluid, it is quite tempting to develop a similar theory for continuous potentials. The Enskog-type theory for the fluids with the continuous potentials should also be useful to calculate the time-dependent memory kernels whose integration over time provides the transport coefficients. For the hardsphere fluid, the memory kernel is always a $\delta$ function in time under the Enskog approximation because of the instantaneous nature of the binary collisions. For fluids with continuous potentials, however, it should decay within nonzero time. The Enskog-type theory should prove to be a very good approximation to describe the short-time behavior of the memory kernel because the binary collisions are dominant dynamical processes at short times. This fact is very important to evaluate the transport coefficient in the high density region under the MCT scheme where the knowledge of the short-time dynamics is indispensable. The short-time dynamics is important also in many dynamical processes in liquids, such as vibrational energy relaxation, barrier crossing dynamics. ${ }^{7}$ Unfortunately, the short-time dynamics depends 
critically on the details of the intermolecular potential. So far, ad hoc fitting functions such as the Gaussian approximation have been used. ${ }^{6,8}$ While the Gaussian approximation is satisfactory at very high densities, it fails miserably at low and intermediate densities. Enskog-type theory is expected to provide a much better understanding of the dynamics without any ad hoc parameter.

There have already been several attempts to develop the Enskog theory for the continuous potentials. Leegwater ${ }^{9}$ was perhaps the first to develop an Enskog-type kinetic theory to analyze the velocity autocorrelation function for an atom in Lennard-Jones fluid. His result reduces to the ChapmanEnskog result in the low density limit but does not satisfy the initial value of the memory kernel for the denser region. Later Pathak et al. developed the Enskog-type theory for the Lennard-Jones fluid starting from the Green-Kubo formula and calculated the velocity correlation function and the selfdiffusion coefficient, ${ }^{10}$ the longitudinal current correlation function, ${ }^{11}$ and the transverse current correlation function. ${ }^{12}$ They argued that the quantities which they calculated are the time correlation functions rather than the memory kernels and, therefore, the expressions they derived are no more than an approximation of the Enskog theory. Furthermore, the transport coefficients which should be obtained by integrating the memory kernels over time are not easy to calculate by their method. The relationship of their methods with the conventional Chapman-Enskog theory in the low density limit were left unclear.

In this paper, we develop a concise method to implement the Enskog theory for the memory kernel and the selfdiffusion coefficient of simple fluids with continuous potentials. Here we shall focus only on the Lennard-Jones potential. We start with the Green-Kubo formula and use the above-mentioned assumptions (1) and (2) to derive general expressions for the memory kernel and the self-diffusion coefficient. It is shown that the present theory satisfies all three prerequisite conditions for the theory. (i) It gives the correct initial value for the memory kernel. (ii) The transport coefficient reduces to that for the Chapman-Enskog theory in the low density limit. (iii) For hard-sphere fluid, it gives the well-known Enskog result. Using the expression derived, we evaluate the memory kernel and compare its time dependence with the simulation data. The self-diffusion coefficient is easily calculated by integrating the memory kernel over time. We give systematic comparison with the simulation results and also with the results for the hard-sphere fluid. The density dependence of the ratio of the self-diffusion coefficient evaluated from the molecular dynamics simulation to that derived from the Enskog theory is plotted. For the hardsphere fluid, it is known that the simulation result becomes larger than the Enskog value at the intermediate density region. This is attributed to the presence of the "backflow" effect which is also the origin of the so-called long-time tail. ${ }^{2,3}$ One may expect that this could also be true for fluids with other potentials since this backflow effect is due to the long-ranged correlated collisions which should be insensitive to the microscopic nature of the binary collisions. We show that, at least quantitatively, the backflow effect is negligible for the Lennard-Jones fluid.
This paper is organized as follows. In Sec. II, the formulation is elucidated. The relation to the Chapman-Enskog theory is also discussed here. In Sec. III, the time-dependent memory kernel is calculated for the Lennard-Jones potential. The comparison with simulation results available is given. A closed formula to calculate the self-diffusion coefficient is given in Sec. IV. The comparison with the simulation results are systematically given here. Section V is devoted to conclusions.

\section{GENERAL FORMULATION}

Consider an atom in a simple fluid which interacts with other identical atoms with a pairwise potential $\phi(r)$. The motion of an atom in a gas or a liquid is often described in terms of the velocity autocorrelation function, $\psi(t)$ $=\langle\mathbf{v}(t) \cdot \mathbf{v}(0)\rangle$, where $\mathbf{v}(t)$ is the velocity of the atom at time $t$ and $\langle\cdots\rangle$ is the equilibrium ensemble average. Its time evolution is well described by a generalized Langevin equation. ${ }^{4}$ If one takes Laplace transform in time, this equation is written as

$$
[m z+\zeta(z)] \widetilde{\psi}(z)=\psi(t=0),
$$

where $m$ is the mass of the atom, $\widetilde{\psi}(z)$ denotes the Laplace transformation of $\psi(t)$, and $\zeta(z)$ is the frequency-dependent friction coefficient or the memory kernel. The memory kernel is given by the Green-Kubo formula as ${ }^{4}$

$$
\zeta(z)=\frac{1}{3 k_{B} T} \int_{0}^{\infty} d t e^{-z t}\langle\mathbf{F}(t) \cdot \mathbf{F}(0)\rangle .
$$

Here $\mathbf{F}(t)$ is the force between the solute and surrounding solvents at a time $t, k_{B}$ is the Boltzmann constant, and $T$ is the absolute temperature. Note that the integrand in Eq. (2) is not the usual force-force time correlation function. The time evolution of $\mathbf{F}(t)$ is not driven by the usual Liouville operator but given by

$$
\mathbf{F}(t) \equiv \exp \left[i \mathcal{Q} L_{N} t\right] \mathbf{F}(0),
$$

where $i L_{N}$ is the Liouville operator for the $N$-particle system and $\mathcal{Q}=1-\mathcal{P}$ and $\mathcal{P}$ is a projection operator defined by $\mathcal{P} *$ $=\left\langle * v_{x}\right\rangle v_{x} /\left\langle v_{x}^{2}\right\rangle$. This equation can be established by using the Mori-Zwanzig projection-operator method. ${ }^{4,13,14}$ The self-diffusion coefficient $D$ is related to the friction coefficient $\zeta$ via the Einstein relation as

$$
D=\frac{k_{B} T}{\zeta}
$$

where $\zeta \equiv \zeta(z=0)$ is the friction coefficient in the stationary limit. Equation (2) can be rewritten in terms of the two particle phase-space distribution function

$$
f_{2}(1,2) \equiv \delta\left(1-q_{1}\right) \sum_{i=2}^{N} \delta\left(2-q_{i}\right)
$$

where $1=\left(\mathbf{r}_{1}, \mathbf{p}_{1}\right)$ and $q_{i}=\left(\mathbf{R}_{i}, \mathbf{P}_{i}\right) \quad(i=1,2, \ldots, N)$ are the phase-space coordinates and the actual coordinates of the particles, respectively. Using this distribution function, $\mathbf{F}(t)$ may be rewritten as 


$$
\mathbf{F}(t)=-\int d 1 d 2 e^{i \mathcal{Q L} L_{N} t} f_{2}(1,2) \nabla_{1} \phi\left(r_{12}\right),
$$

where $r_{12}=\left|\mathbf{r}_{1}-\mathbf{r}_{2}\right|$. Equation (2) can be rewritten, using this expression, as

$$
\begin{aligned}
\zeta(z)= & \frac{1}{3 V k_{B} T} \sum_{\alpha=x, y, z} \int d 1 d 2 d 3 d 4 \frac{\partial \phi\left(r_{12}\right)}{\partial r_{1 \alpha}} \\
& \times G(1,2 ; 3,4 ; z) \frac{\partial \phi\left(r_{34}\right)}{\partial r_{3 \alpha}},
\end{aligned}
$$

where $V$ is the volume of the system and $G(1,2 ; 3,4 ; z)$ $=V\left\langle\left\{\left(z-i \mathcal{Q} L_{N}\right)^{-1} f_{2}(1,2)\right\} f_{2}(3,4)\right\rangle$ is the four points propagator which describes the time evolution of a particle interacting with other particles. ${ }^{6,8}$ This representation makes it easy to understand how the binary collision approximation comes to play a role. Mazenko ${ }^{15}$ showed that the equation for $G(1,2 ; 3,4 ; z)$ can be expressed without invoking the projected Liouville operator $i \mathcal{Q} L_{N}$ by introducing the phase-space correlation functions and is given by

$$
\begin{aligned}
& {\left[z+i L_{2}(1,2)\right] G(1,2 ; 3,4 ; z)+\int d 1^{\prime} d 2^{\prime} d 3^{\prime} \widetilde{C}\left(1,2 ; 3^{\prime}\right) \widetilde{C}^{-1}\left(3^{\prime} ; 1^{\prime}\right) i L_{2 I}\left(1^{\prime}, 2^{\prime}\right) G\left(1^{\prime}, 2^{\prime} ; 3,4 ; z\right)} \\
& \quad+\int d 1^{\prime}\left\{i L_{2 I}\left(1,1^{\prime}\right)+i L_{2 I}\left(2,1^{\prime}\right)\right\}\left[C\left(1,2,1^{\prime} ; 3,4 ; z\right)-\int d 2^{\prime} d 3^{\prime} C\left(1,2,1^{\prime} ; 2^{\prime} ; z\right) \widetilde{C}^{-1}\left(2^{\prime} ; 3^{\prime}\right) C\left(3^{\prime} ; 3,4 ; z\right)\right]=\widetilde{G}(1,2 ; 3,4)
\end{aligned}
$$

where

$$
\begin{aligned}
& i L_{2}(1,2)=\frac{\mathbf{p}_{1}}{m} \cdot \nabla_{1}+\frac{\mathbf{p}_{2}}{m} \cdot \nabla_{2}-\frac{\partial \phi\left(r_{12}\right)}{\partial \mathbf{r}_{1}} \cdot\left(\frac{\partial}{\partial \mathbf{p}_{1}}-\frac{\partial}{\partial \mathbf{p}_{2}}\right), \\
& i L_{2 I}(1,2)=-\frac{\partial \phi\left(r_{12}\right)}{\partial \mathbf{r}_{1}} \cdot\left(\frac{\partial}{\partial \mathbf{p}_{1}}-\frac{\partial}{\partial \mathbf{p}_{2}}\right)
\end{aligned}
$$

are the Liouville operator for the two-body system and its interaction part, respectively. In this expression, $C\left(1,2, \ldots ; 1^{\prime}, 2^{\prime}, \ldots ; z\right)$ is the phase-space correlation function. For example, $C\left(1,2,3 ; 1^{\prime}, 2^{\prime} ; z\right)$ is defined by $V\langle\{(z$ $\left.\left.\left.+i L_{N}\right)^{-1} f_{3}(1,2,3)\right\} f_{2}\left(1^{\prime}, 2^{\prime}\right)\right\rangle$, where

$$
f_{3}(1,2,3)=\delta\left(1-q_{1}\right) \sum_{i \neq j}^{N} \delta\left(2-q_{i}\right) \delta\left(3-q_{j}\right)
$$

is the three-particle phase-space distribution function. The other correlation functions are defined in the similar manner. The quantities with a tilde represent initial-time values of them and $\widetilde{C}^{-1}$, s are their inverse. One can see that all but the first term on the left-hand side of Eq. (7) involve more than three particle interactions. Therefore, under the binary collision approximation (on which the idea of the Boltzmann equation is based), we may disregard all these terms. Therefore, the friction coefficient under the binary collision approximation, which we shall refer to as $\zeta_{E}(z)$, can be written as

$$
\begin{aligned}
\zeta_{E}(z)= & \frac{1}{3 V k_{B} T} \sum_{\alpha=x, y, z} \int d 1 d 2 d 3 d 4 \frac{\partial \phi\left(r_{12}\right)}{\partial r_{1 \alpha}} \\
& \times\left[z+i L_{2}(1,2)\right]^{-1} \widetilde{G}(1,2 ; 3,4) \frac{\partial \phi\left(r_{34}\right)}{\partial r_{3 \alpha}} .
\end{aligned}
$$

Furthermore, one may use the following identity:

$$
\begin{aligned}
& \int d 3 d 4 \widetilde{G}(1,2 ; 3,4) \frac{\partial \phi\left(r_{34}\right)}{\partial r_{3 \alpha}} \\
&=-\rho k_{B} T f_{0}\left(p_{1}\right) f_{0}\left(p_{2}\right) \frac{\partial g\left(r_{12}\right)}{\partial r_{1 \alpha}},
\end{aligned}
$$

where $f_{0}(p)=\left(2 \pi m k_{B} T\right)^{-3 / 2} \exp \left[-p^{2} / 2 m k_{B} T\right]$ is the Maxwell distribution function, $\rho$ is the number density of the system, and $g(r)$ is the radial distribution function. Then, we may simplify the expression as

$$
\begin{aligned}
\zeta_{E}(z)= & -\frac{\rho}{3 V} \sum_{\alpha=x, y, z} \int d 1 d 2 \frac{\partial \phi\left(r_{12}\right)}{\partial r_{1 \alpha}} \\
& \times\left[z+i L_{2}(1,2)\right]^{-1} f_{0}\left(p_{1}\right) f_{0}\left(p_{2}\right) \frac{\partial g\left(r_{12}\right)}{\partial r_{1 \alpha}} \\
= & -\frac{\rho}{3 V} \sum_{\alpha=x, y, z} \int d 1 d 2 f_{0}\left(p_{1}\right) f_{0}\left(p_{2}\right) \frac{\partial g\left(r_{12}\right)}{\partial r_{1 \alpha}} \\
& \times\left[z-i L_{2}(1,2)\right]^{-1} \frac{\partial \phi\left(r_{12}\right)}{\partial r_{1 \alpha}} .
\end{aligned}
$$

Note that, in Eq. (11), the time evolution operator acts only on the bare potential term. As one is calculating the binary contribution to the friction, $g(r)$ is not involved with the time evolution. $g(r)$ controls an initial configuration for a pair of atoms before a collision. After $g(r)$ decides the initial configuration, the time evolution of the pair (or the force acting on it) would be driven by the two-particle Liouville operator.

This equation can be further simplified by introducing the center-of-mass and relative coordinates; 


$$
\begin{aligned}
& \mathbf{r}=\mathbf{r}_{1}-\mathbf{r}_{2}, \quad \mathbf{R}=\frac{1}{2}\left(\mathbf{r}_{1}+\mathbf{r}_{2}\right), \\
& \mathbf{p}=\frac{1}{2}\left(\mathbf{p}_{1}-\mathbf{p}_{2}\right), \quad \mathbf{P}=\mathbf{p}_{1}+\mathbf{p}_{2} .
\end{aligned}
$$

The Liouville operator in this representation can be written as

$$
\begin{aligned}
i L_{2}(1,2) & =\frac{\mathbf{P}}{2 m} \cdot \frac{\partial}{\partial \mathbf{R}}+\frac{2 \mathbf{p}}{m} \cdot \frac{\partial}{\partial \mathbf{r}}-\frac{\partial \phi(r)}{\partial \mathbf{r}} \cdot \frac{\partial}{\partial \mathbf{p}} \\
& \equiv \frac{\mathbf{P}}{2 m} \cdot \frac{\partial}{\partial \mathbf{R}}+i L(\mathbf{r}, \mathbf{p}) .
\end{aligned}
$$

Using this representation, Eq. (11) can be written as

$$
\begin{aligned}
\zeta_{E}(z)= & -\frac{\rho}{3 V} \sum_{\alpha=x, y, z} \int d \mathbf{P} \int d \mathbf{p} \int d \mathbf{R} \\
& \times \int d \mathbf{r} f_{0}^{\mathrm{tot}}(P) f_{0}^{\mathrm{red}}(p) \frac{\partial g(r)}{\partial r_{\alpha}}[z-i L(\mathbf{r}, \mathbf{p})]^{-1} \frac{\partial \phi(r)}{\partial r_{\alpha}},
\end{aligned}
$$

where $f_{0}^{\mathrm{tot}}(P)=\left(4 \pi m k_{B} T\right)^{-3 / 2} \exp \left[-P^{2} / 4 m k_{B} T\right]$ and $f_{0}^{\mathrm{red}}(p)$ $=\left(\pi m k_{B} T\right)^{-3 / 2} \exp \left[-p^{2} / m k_{B} T\right]$. Integrating over $\mathbf{P}$ and $\mathbf{R}$, we arrive at

$$
\begin{aligned}
\zeta_{E}(z)= & -\frac{\rho}{3} \sum_{\alpha=x, y, z} \int d \mathbf{p} \int d \mathbf{r} f_{0}^{\mathrm{red}}(p) \frac{\partial g(r)}{\partial r_{\alpha}} \\
& \times[z-i L(\mathbf{r}, \mathbf{p})]^{-1} \frac{\partial \phi(r)}{\partial r_{\alpha}} .
\end{aligned}
$$

Or in the real time representation, we have

$$
\begin{aligned}
\zeta_{E}(t)= & -\frac{\rho}{3} \sum_{\alpha=x, y, z} \int d \mathbf{p} \int d \mathbf{r} f_{0}^{\mathrm{red}}(p) \frac{\partial g(r)}{\partial r_{\alpha}} \\
& \times \exp [i L(\mathbf{r}, \mathbf{p}) t] \frac{\partial \phi(r)}{\partial r_{\alpha}} .
\end{aligned}
$$

Equations (15) and (16) are the Enskog expressions for the frequency- and time-dependent memory kernels, respectively, and are the primary results of this paper.

Pathak et al. ${ }^{10}$ presented the same expressions as these equations but they argued that these equations are just forceforce time correlation functions and no more than an approximation to the "exact" Enskog theory. As is clear from our derivation, this is not the time force-force correlation but the memory kernel itself. The irreversible nature of the memory kernel came in when the binary operator was introduced. This procedure corresponds to the truncation of the
Bogolyubov-Born-Green-Kirkwood-Yvon hierarchy up to the lowest level. ${ }^{1}$ And Eq. (11) clearly shows that the velocity distributions of the two particles before the binary collision are independent (the molecular chaos assumption).

From Eqs. (15) and (16), we may prove the following three important properties well known in the kinetic theory of fluids.

(i) Equation (16) gives the exact initial value for arbitrary density.

$$
\begin{aligned}
\zeta_{E}(t=0) & =-\frac{\rho}{3} \sum_{\alpha=x, y, z} \int d \mathbf{p} \int d \mathbf{r} f_{0}^{\mathrm{red}}(p) \frac{\partial g(r)}{\partial r_{\alpha}} \frac{\partial \phi(r)}{\partial r_{\alpha}} \\
& =\frac{\rho}{3} \int d \mathbf{r} g(r) \nabla^{2} \phi(r),
\end{aligned}
$$

which is the well-known expression for the Einstein frequency. This was first shown by Pathak et al. ${ }^{10}$

(ii) Equation (15) has been derived from the GreenKubo formula. This expression in the stationary limit $(z$ $=0$ ) looks very different from the collision integral derived from Chapman-Enskog theory which is familiar in the textbooks of the kinetic theory. ${ }^{16-18}$ We show now that it is indeed equivalent to the result from the conventional theory in the low density limit. In this limit, the radial distribution function is given by $g(r) \simeq e^{-\phi(r) / k_{B} T}$ and the memory kernel [we now refer to as $\zeta_{B}(z)$ ] of Eq. (15) is written as

$$
\begin{aligned}
\zeta_{B}(z)= & \frac{\rho}{3 k_{B} T} \sum_{\alpha=x, y, z} \int d \mathbf{p} \int d \mathbf{r} f_{0}^{\mathrm{red}}(p) e^{-\phi(r) / k_{B} T} \frac{\partial \phi(r)}{\partial r_{\alpha}} \\
& \times[z-i L(\mathbf{r}, \mathbf{p})]^{-1} \frac{\partial \phi(r)}{\partial r_{\alpha}} .
\end{aligned}
$$

Using the following identities,

$$
\begin{aligned}
& -\frac{\partial \phi(r)}{\partial r_{\alpha}}=i L_{I}(\mathbf{r}, \mathbf{p}) p_{\alpha}, \\
& i L_{I} \frac{1}{z-i L}=-1+\left(z-i L_{0}\right) \frac{1}{z-i L},
\end{aligned}
$$

where

$$
i L_{0}(\mathbf{r}, \mathbf{p})=\frac{2 \mathbf{p}}{m} \cdot \frac{\partial}{\partial \mathbf{r}}, \quad i L_{I}(\mathbf{r}, \mathbf{p})=-\frac{\partial \phi(r)}{\partial \mathbf{r}} \cdot \frac{\partial}{\partial \mathbf{p}}
$$

are the kinetic and the potential part of the Liouville operator, respectively, Eq. (18) can be rewritten after several partial integrations as

$$
\begin{aligned}
\zeta_{B}(z) & =\frac{\rho}{3 k_{B} T} \sum_{\alpha=x, y, z} \int d \mathbf{p} \int d \mathbf{r} p_{\alpha} i L_{I}(\mathbf{r}, \mathbf{p}) f_{0}^{\mathrm{red}}(p) e^{-\phi(r) / k_{B} T}[z-i L(\mathbf{r}, \mathbf{p})]^{-1} \frac{\partial \phi(r)}{\partial r_{\alpha}} \\
& =\frac{\rho}{3 k_{B} T} \sum_{\alpha=x, y, z} \int d \mathbf{p} \int d \mathbf{r} p_{\alpha}\left\{z-i L_{0}(\mathbf{r}, \mathbf{p})\right\} f_{0}^{\mathrm{red}}(p) e^{-\phi(r) / k_{B} T}[z-i L(\mathbf{r}, \mathbf{p})]^{-1} \frac{\partial \phi(r)}{\partial r_{\alpha}} \\
& =-\frac{\rho}{3 k_{B} T} \sum_{\alpha=x, y, z} \int d \mathbf{p} \int d \mathbf{r} p_{\alpha}\left\{z-i L_{0}(\mathbf{r}, \mathbf{p})\right\} f_{0}^{\mathrm{red}}(p) e^{-\phi(r) / k_{B} T} \int_{0}^{\infty} d t e^{-z t} \frac{d p_{\alpha}(t)}{d t},
\end{aligned}
$$


where $p_{\alpha}(t)=e^{i L(\mathbf{r}, \mathbf{p}) t} p_{\alpha}$. One can integrate Eq. (19) over time for the stationary limit, $\zeta_{B}=\zeta_{B}(z=0)$, as

$$
\begin{aligned}
\zeta_{B}= & \frac{\rho}{3 k_{B} T} \int d \mathbf{p} \int d \mathbf{r} i L_{0}(\mathbf{r}, \mathbf{p}) f_{0}^{\mathrm{red}}(p) e^{-\phi(r) / k_{B} T} \\
& \times\left\{\mathbf{p} \cdot \mathbf{p}(t=\infty)-p^{2}\right\} .
\end{aligned}
$$

In the integration over $\mathbf{r}$, we shall use the cylindrical coordinate and we choose the axial direction along the direction of the momentum,

$$
\begin{aligned}
\zeta_{B}= & \frac{\rho}{3 k_{B} T} \int d \mathbf{p} \int_{-\infty}^{\infty} d h \int_{0}^{\infty} b d b \int_{0}^{2 \pi} d \theta \frac{2 p}{m} \frac{\partial}{\partial h} \\
& \times f_{0}^{\mathrm{red}}(p) e^{-\phi(r) / k_{B} T}\left\{\mathbf{p} \cdot \mathbf{p}(t=\infty)-p^{2}\right\} \\
= & \frac{16 \pi^{2} \rho}{3 m k_{B} T} \int_{0}^{\infty} d p \int_{0}^{\infty} b d b p^{5} f_{0}^{\mathrm{red}}(p)\{\cos \chi(p, h=\infty, b) \\
& -\cos \chi(p, h=-\infty, b)\},
\end{aligned}
$$

where $\chi(p, h, b)$ is the deflection angle of the binary collision for the particle which initially had the momentum $p$ along the $h$ direction and was at the position $(h, b)$ in the cylindrical coordinate. In the derivation we have used the momentum conservation at $t=0$ and $t=\infty$, at which the particles are far apart from the collision center. Since the first term in the integrand represents the motion of the particle which initially lies at $h=\infty$ and goes apart from the collision center, we have $\chi(p, h=\infty, b)=0$. On the other hand, the second term is a function of both $p$ and $b$. Representing $\chi(p, h=-\infty, b)$ as $\chi(p, b)$. Equation (21) is reduced to

$$
\zeta_{B}=\frac{8}{3} \rho \sigma^{2} \sqrt{m \pi k_{B} T} \Omega^{(1,1) *}(T)
$$

with the collision integral given by

$$
\begin{aligned}
\Omega^{(1,1) *}(T) \equiv & \frac{2}{\sigma^{2}\left(m k_{B} T\right)^{3}} \int_{0}^{\infty} d p \int_{0}^{\infty} b d b p^{5} e^{-p^{2} / m k_{B} T} \\
& \times\{1-\cos \chi(b, p)\} .
\end{aligned}
$$

The deflection angle $\chi(b, p)$ is given by solving the equation of motion for this two-particle system ${ }^{1}$ as

$$
\chi(b, p)=\pi-2 b \int_{r_{\min }}^{\infty} d r \frac{1}{r^{2} \sqrt{1-\frac{m \phi(r)}{p^{2}}-\frac{b^{2}}{r^{2}}}} .
$$

Equation (23) is exactly the expression for the collision integral well known for several decades.

(iii) The Enskog value for the friction for the hardsphere fluid is known to be given by

$$
\zeta_{E, \mathrm{HS}}=\frac{8}{3} \rho \sigma^{2} g(\sigma) \sqrt{m \pi k_{B} T},
$$

where $\sigma$ is the radius of the sphere. It is straightforward to show that our expression reduces to this result. First, we introduce a function $y(r)$ defined by $g(r)=y(r) e^{-\phi(r) / k_{B} T}$. $y(r)$ is a continuous and smooth function. ${ }^{5}$ Using the fact that $e^{-\phi(r) / k_{B} T}$ is the Heaviside function, Eq. (15) can be written as

$$
\begin{aligned}
\zeta_{E, \mathrm{HS}}(z)= & -\frac{\rho}{3} \int d \mathbf{p} \int d \mathbf{r} f_{0}^{\mathrm{red}}(p)\left\{y^{\prime}(r) e^{-\phi(r) / k_{B} T}\right. \\
& +g(\sigma) \delta(r-\sigma)\}[z-i L(\mathbf{r}, \mathbf{p})]^{-1} \frac{d \phi(r)}{d r} .
\end{aligned}
$$

We neglect the first term in the parentheses of Eq. (26), assuming that the contribution from the slow varying $y^{\prime}(r)$ is smaller than that from the sharply peaked delta function. Therefore, we may write Eq. (26) as

$$
\begin{aligned}
\zeta_{E, \mathrm{HS}}(z)= & -\frac{\rho g(\sigma)}{3} \sum_{\alpha=x, y, z} \int d \mathbf{p} \int d \mathbf{r} f_{0}^{\mathrm{red}}(p) \\
& \times\left\{\frac{\partial}{\partial r_{\alpha}} e^{-\phi(r) / k_{B} T}\right\}[z-i L(\mathbf{r}, \mathbf{p})]^{-1} \frac{\partial \phi(r)}{\partial r_{\alpha}} .
\end{aligned}
$$

Except for the factor of $g(\sigma)$, this is the same as Eq. (18) which we showed to be equivalent to the Chapman-Enskog expression in the previous paragraph. For the hard-sphere fluid, this is known to be given by $8 \rho \sigma^{2} \sqrt{m \pi k_{B} T} / 3$ for $z$ $=0$ and we immediately arrive at Eq. (25).

\section{THE MEMORY KERNEL}

In this section, we present numerical calculations of the time-dependent Enskog memory kernel, Eq. (16). Hereafter, we shall focus only on the Lennard-Jones potential defined by

$$
\phi(r)=4 \epsilon\left\{\left(\frac{\sigma}{r}\right)^{12}-\left(\frac{\sigma}{r}\right)^{6}\right\},
$$

where $\epsilon$ and $\sigma$ are the standard Lennard-Jones parameters. Basically, one may evaluate the memory kernel provided that the positions of all possible trajectories of the two particle system are known. This can be done easily by solving the equation of motion. We evaluate the positions $(r(t), \theta(t))$ at time $t$ in the polar coordinates for a given initial condition $(p, r, \theta)$, where we choose the direction of the velocity parallel to the polar axis. The problem can be reduced to the one-dimensional problem of the motion in the effective potential given by $\phi_{\text {eff }}(r)=\phi(r)+p^{2} b^{2} / m r^{2}$, where $b \equiv r \sin \theta$ is the impact parameter which represents the perpendicular distance between the collision center and the initial position. ${ }^{19}$ The solution of the equation of motion is given by

$$
t=\int^{r(t)} d r f(r), \quad \theta(t)=\frac{2 b p}{m} \int^{r(t)} d r \frac{f(r)}{r^{2}},
$$

where $f(r)=1 / \sqrt{4\left\{E-\phi_{\text {eff }}(r)\right\} / m} . \quad r(t)$ for arbitrary $t$ is evaluated by solving the first equation of Eq. (29). $\theta(t)$ can be evaluated from the second equation using $r(t)$ evaluated from the first. The constants of integration can be chosen such that they satisfy the initial condition. For the LennardJones potential, the motion in this effective potential may have three turning points at most, depending on the initial conditions. Radius of turning points are given by the zeros of $E-\phi_{\text {eff }}(r)=0$, where $E$ is the total energy of the two particle system. Note that if the initial radius is smaller than the largest turning point, some trajectories form the bounded trajectories. We incorporated all possible trajectories in order to evaluate Eq. (16). $g(r)$ was calculated using the Percus- 

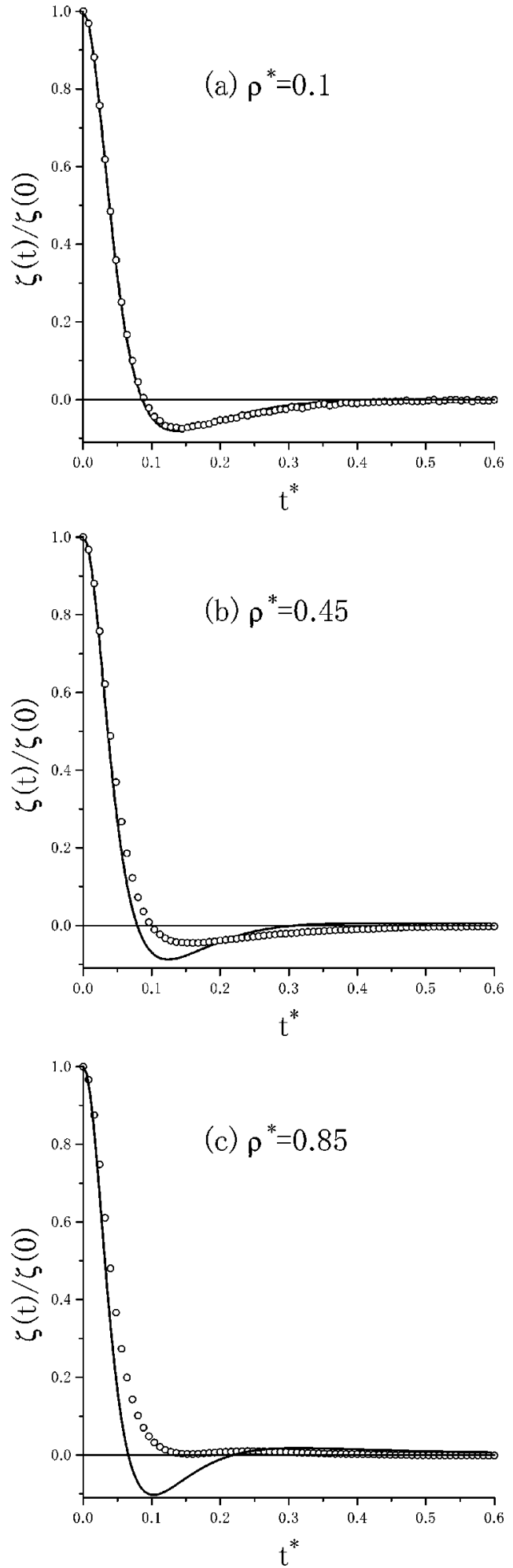

FIG. 1. $\zeta_{E}(t) / \zeta_{E}(0)$ for $T^{*}=1.5$, where $t^{*}=t / \sqrt{m \sigma^{2} / \epsilon}$. (a) for $\rho^{*}=0.1$, (b) for $\rho^{*}=0.45$, (c) for $\rho^{*}=0.85$. The open circles are the molecular dynamics simulation results given by Yamguchi et al. (Ref. 21).

Yevick closure with the Ornstein-Zernike relation ${ }^{5,16}$ for $\rho^{*}<0.45$, where $\rho^{*}=\rho \sigma^{3}$ is the dimensionless density. For higher density, computer simulations were used. SMSA approximation ${ }^{20}$ was also used to check the result. The result for $\rho^{*}=0.1,0.45$, and 0.85 at $T^{*}=1.5$ is given in Fig. 1 along with the molecular dynamics simulation given by
Yamaguchi et al. ${ }^{21}$ Here $T^{*}=k_{B} T / \epsilon$ is the dimensionless temperature. The results are normalized by their initial values. We have also checked that the initial values for various densities agree well with the simulation results. ${ }^{22}$ The agreement with the simulation result is excellent for $\rho^{*}=0.1$. One observes that the initial fast decay is followed by a negative tail. This negative dip is due to the attractive part of the potential; most of the initial positions are lying in the attractive part of the potential and a half of the initial velocity is the inward to the collision center and another half is going outward. The particle going inward toward the collision center hits the very steep repulsive part of the potential which changes the sign of the force (from that at the initial time), whereas the force for the particle going outward will remain positive but it is much smaller in magnitude. For higher densities, the agreement is poor, as expected. The simulation results show that the depth of negative dip gets smaller as the density increases, whereas the present Enskog results show the opposite trend. The larger Enskog negative tail at higher densities originates from the increase in the probability of the particle sitting around the first peak of $g(r)$ which leads to a bigger chance for the particle to hit the repulsive wall.

The above-mentioned observation may be rationalized as follows. One possible reason is that the effect of the correlated collisions effect (or the mode-coupling effect) is very sensitive to the shape of potential. The first pronounced effect of the correlated collisions for the hard-sphere fluid as the density increases is the backflow effect due to the transverse current mode $2,3,23,24$ which leads to the so-called longtime-tail. This tail is always negative. As the density reaches the liquid region, the positive tail due to the density fluctuation mode overcomes the backflow effect. ${ }^{25}$ The monotonic decrease of amplitudes observed in the simulation for the Lennard-Jones fluid might indicate that the backflow effect is too small to enhance the negative tail and the density fluctuations always play a more dominant role for this system. To confirm the presence of the backflow effect, one needs to calculate the memory kernel for longer time where both the binary and density mode contributions disappear and only the current mode survives.

Another possible explanation of the vanishing negative tail might be the presence of multiple collisions. The collisions in the continuous potentials are not simultaneous and the third or fourth particles can influence the binary collision. This might encourage the particle trapped in the attractive dip of the Lennard-Jones potential to escape from it and, therefore, reduce the amplitude of the negative dip. This effect cannot be taken into account either in the Enskog theory or in the mode-coupling theory in which only the binary and the ring collision terms are taken into account. We shall come back to this point in Sec. IV where the argument on the contribution of the different modes to the self-diffusion coefficient is given.

\section{ENSKOG THEORY FOR $D_{E}$}

In this section, we derive expressions of the friction coefficient and the self-diffusion coefficient by integrating the memory kernel over time. The steady state value of $\zeta_{E}$ can be obtained by setting $z=0$ in Eq. (15) as 


$$
\begin{aligned}
\zeta_{E} & =\frac{\rho}{3} \sum_{\alpha=x, y, z} \int_{0}^{\infty} d t \int d \mathbf{p} \int d \mathbf{r} f_{0}^{\mathrm{red}}(p) \frac{\partial g(r)}{\partial r_{\alpha}} \frac{d p_{\alpha}(t)}{d t} \\
& =\frac{\rho}{3} \sum_{\alpha=x, y, z} \int d \mathbf{p} \int d \mathbf{r} f_{0}^{\mathrm{red}}(p) \frac{\partial g(r)}{\partial r_{\alpha}}\left\{p_{\alpha}(t=\infty)-p_{\alpha}\right\} .
\end{aligned}
$$

We may again use the cylindrical coordinate for $\mathbf{r}$ $=(h, b, \theta)$ and choose the direction of the $h$ axis parallel to p. $b$ is the impact parameter. Since the system considered is isotropic, we have

$$
\begin{aligned}
\zeta_{E}= & \frac{\rho}{3} \sum_{\alpha=x, y, z} \int d \mathbf{p} \int_{-\infty}^{\infty} d h \int_{0}^{\infty} b d b \int_{0}^{2 \pi} d \theta f_{0}^{\mathrm{red}}(p) \\
& \times\left\{\hat{p}_{\alpha} \frac{\partial g(r)}{\partial h}+\hat{e}_{1, \alpha} \frac{\partial g(r)}{\partial b}+\hat{e}_{2, \alpha} \frac{1}{b} \frac{\partial g(r)}{\partial \theta}\right\} \\
& \times\left\{p_{\alpha}(t=\infty)-p_{\alpha}\right\}, \\
= & \frac{\rho}{3} \int d \mathbf{p} \int_{-\infty}^{\infty} d h \int_{0}^{\infty} b d b \int_{0}^{2 \pi} d \theta f_{0}^{\mathrm{red}}(p) \\
& \times\left[\frac{\partial g(r)}{\partial h}\{\hat{\mathbf{p}} \cdot \mathbf{p}(t=\infty)-p\}+\frac{\partial g(r)}{\partial b} \hat{\mathbf{e}}_{1} \cdot \mathbf{p}(t=\infty)\right],
\end{aligned}
$$

where $\hat{\mathbf{p}}$ is the unit vector of the momentum, and $\hat{\mathbf{e}}_{1}, \hat{\mathbf{e}}_{2}$ are the two unit vectors perpendicular to $\hat{\mathbf{p}}$, and $r=\sqrt{h^{2}+b^{2}}$ is the distance from the collision center. Depending on the initial momenta and positions, some trajectories do not go apart from the collision center but they form stable orbital trajectories. Curtis ${ }^{26}$ discussed the effect of such bounded trajectories on the friction coefficient in the low density limit for the Lennard-Jones (LJ) fluid. He concluded that at sufficiently high temperature (above $T^{*}>1.0$ ), this effect is very small. Therefore, we shall disregard these bounded trajectories in our calculations. For unbounded trajectories, we can write $\quad \hat{\mathbf{p}} \cdot \mathbf{p}(t=\infty)=p_{\infty} \cos \chi(p, b, h) \quad$ and $\quad \hat{\mathbf{e}}_{1} \cdot \mathbf{p}(t=\infty)$ $=p_{\infty} \sin \chi(p, b, h)$, where $\chi(p, b, h)$ is the deflection angle and $p_{\infty}$ is the intensity of the momentum at $t=\infty$ and given from the energy conservation by $p_{\infty}=\sqrt{p^{2}+m \phi(r)}$. Thus, we arrive at the following expression for $\zeta_{E}$,

$$
\zeta_{E}=\frac{8}{3} \rho \sigma^{2} \sqrt{m \pi k_{B} T} \Omega^{(1,1) *}(T)
$$

with the Enskog collision integral $\Omega^{(1,1) *}(T)$ given by

$$
\begin{aligned}
\Omega^{(1,1) *(T)=} & \frac{1}{\left(m k_{B} T \sigma\right)^{2}} \int_{0}^{\infty} d p \int_{0}^{\infty} r^{2} d r \int_{0}^{\pi / 2} \sin \theta d \theta p_{\infty} p^{2} \\
& \times e^{-p^{2} / m k_{B} T} \frac{d g(r)}{d r}\{\cos [\chi(p, h, b)-\theta] \\
& -\cos [\chi(p,-h, b)+\theta]\}
\end{aligned}
$$

where $h=r \cos \theta$ and $b=r \sin \theta$. The deflection angle $\chi(p$, $\pm h, b)$ can be evaluated from the solution of the equation of motion, Eq. (29), with the initial condition $(p, r, \theta)$ for the momentum and position, respectively. Since we consider the unbounded trajectories only, $r(t=\infty)=\infty$ for $t=\infty$. The lower boundary of the second equation of Eq. (29) is given

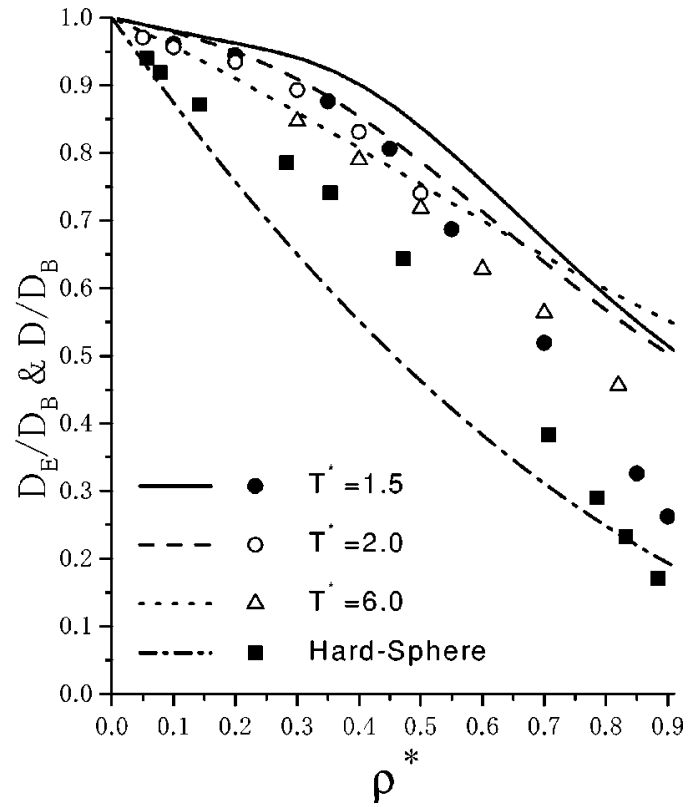

FIG. 2. $D_{E} / D_{B}$ for several temperatures. $\rho^{*}=\rho \sigma^{3}$ is the dimensionless number density. Lines are from the present theory and dots from the molecular dynamics simulation. The solid line and closed circles (Ref. 21) are for $T^{*}=1.5$, the dashed line and open circles (Ref. 27) are for $T^{*}=2.0$, and the dotted line and open triangles are for $T^{*}=6.0$. The hard-sphere Enskog value and simulation result (Ref. 3) are given by the dot-dashed line and closed squares, respectively.

by the turning point, $r_{\min }$, the point where the radial component of the momentum of the particle vanishes, or $d r(t) / d t$ $=0$. This is equivalent to evaluate the zero of $E-\phi_{\text {eff }}(r)$ $=0$. The integration constants are determined in such a way that they satisfy the initial conditions. The expression for deflection angle is different depending on whether the particle is moving toward or away from the collision center and is given explicitly by

$$
\chi(p, b, h)=\left\{\begin{array}{l}
\frac{2 p b}{m} \int_{r_{\min }}^{\infty} d r \frac{f(r)}{r^{2}}+\theta_{\min }+\theta \quad(h<0) \\
\frac{2 p b}{m} \int_{r}^{\infty} d r \frac{f(r)}{r^{2}}+\theta \quad(h \geqslant 0),
\end{array}\right.
$$

where $\theta_{\min }$ is the angle at the turning point and is given by

$$
\theta_{\min }=\frac{2 p b}{m} \int_{r_{\min }}^{r} d r \frac{f(r)}{r^{2}} .
$$

We calculate these integrals for all possible initial conditions and insert them back in to Eq. (32) and integrate it numerically to get the friction coefficient. The Gaussian-quadrature method has been used to implement the multidimensional integration.

The self-diffusion coefficient is calculated from the Einstein relation $D_{E}=k_{B} T / \zeta_{E}$. In Fig. 2, we plot the density dependence of $D_{E} / D_{B}$ for the reduced temperature $T^{*}$ $=1.5,2.0$, and 6.0. Corresponding simulation values are also shown. The molecular dynamics simulation for $T^{*}$ $=1.5$ was done by Yamaguchi et al., ${ }^{21}$ for $T^{*}=2.0$ by Kincaid et al., ${ }^{27}$ and for $T^{*}=6.0$ by the present authors, re- 


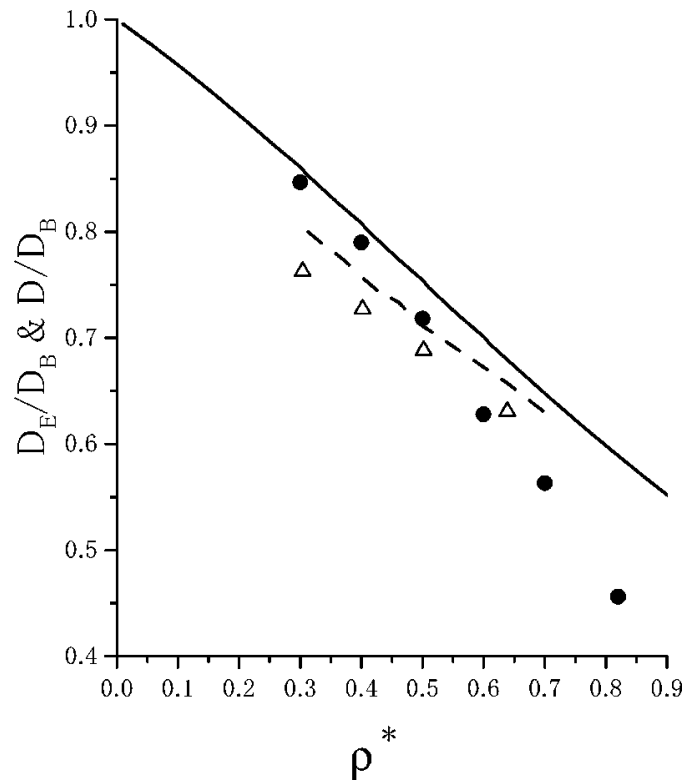

FIG. 3. Comparison of $D_{E} / D_{B}$ at $T^{*}=6.0$ for our theory and simulation with Pathak et al.'s theory and Heyes' simulation. The solid line is from the present theory and the closed circles are from our molecular dynamics simulation. The dashed line is Pathak's theory (Ref. 10) and the open triangles are Heyes' molecular dynamics simulation (Ref. 28).

spectively. For reference, we also show the simulation ${ }^{3}$ and the Enskog result for the hard-sphere fluid. We also checked the results previously given by Pathak et al. ${ }^{10}$ with ours and found that there are considerable differences between the two. They evaluated the self-diffusion coefficient by directly integrating the memory kernel from $t=0$ to $\infty$. Furthermore, they evaluated the trajectories of two particles directly solving the equations of motion which becomes less reliable at long times.

In Fig. 3, we show a comparison between our calculation and that of Pathak et al. at $T^{*}=6.0$. Also in Fig. 3 we show the simulation results of Heyes ${ }^{28}$ and of us. We have carried out molecular dynamics simulations of $500 \mathrm{LJ}$ particles in the $N V E$ ensemble. The reduced time step $0.0005 \tau(\tau$ $\left.=\sqrt{m \sigma^{2} / \epsilon}\right)$ is used for most of the density range. However, we have used much smaller time steps and performed longer simulation runs at very low densities. After $10^{5}$ equilibration steps, simulations have been performed for another $10^{5}$ steps during which the positions and velocities have been stored for subsequent analysis. For each state point three such runs have been carried out and the results reported here are the averages over all three runs. It is clear that the result of Pathak et al. has wrong density dependence at low density where the Enskog theory should be accurate. We found similar deviations from Pathak et al.'s results at low temperatures (not shown) also.

An important point to note is that the deviation of the Enskog values from the Boltzmann values are always smaller for the Lennard-Jones fluid than those for the hardsphere fluid. The density dependence of both simulations and the Enskog theory for the Lennard-Jones fluid are qualitatively the same; the smaller slope for the low density region followed by the steeper decrease in the denser region. As the temperature increases, the slope in the low density gets

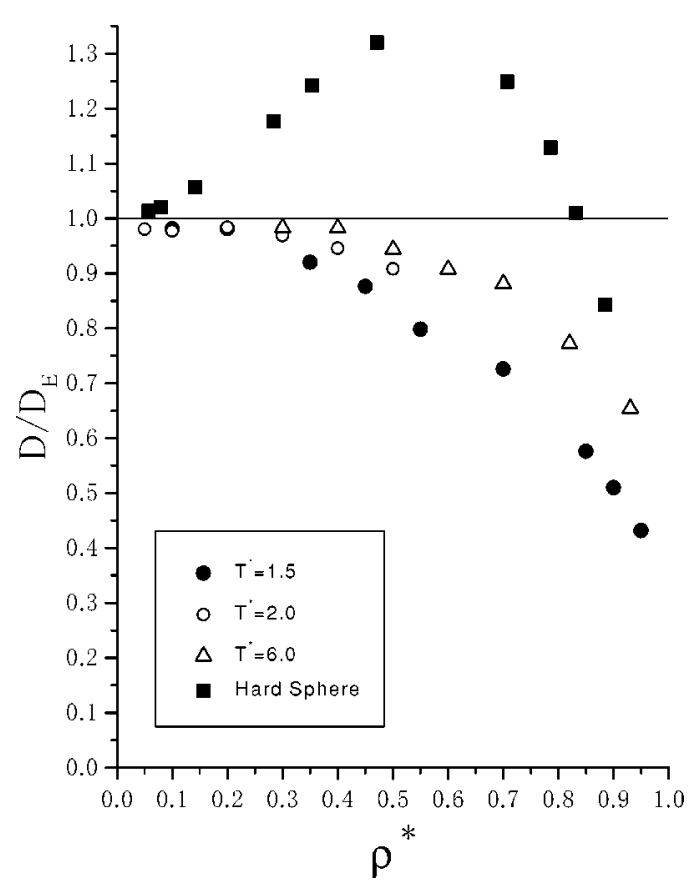

FIG. 4. $D / D_{E}$ for several temperatures. The closed circles are for $T^{*}=1.5$ (Ref. 21), the open circles for $T^{*}=2.0$ (Ref. 27), and the open triangles for $T^{*}=6.0$. The simulation result for hard-sphere fluid (Ref. 3 ) is given by closed squares.

steeper while the opposite trend is observed in the high density region. These tendencies are absent for the hard-sphere fluid. One may rationalize these results as follows. It is known that $g(r)$ of the Lennard-Jones fluid is influenced mostly by the attractive part of the potential in the small density region. As far as the structure in liquid is dominated by the attractive force, $g(r)$ is insensitive to the density. The tendencies are enhanced as the temperature gets lower. This explains the weak dependence of $D_{E} / D_{B}$ on the density at low temperatures, as shown in Fig. 3. On the other hand, as the density increases, the repulsive part starts playing a more important role in $g(r)$. In the repulsion dominated region, the structure becomes more hard-sphere like as the temperature goes down. For the larger temperatures, the potential is effectively "softened" and the excluded volume effect becomes less, which leads to less sensitivity of $g(r)$ on the density. This explains the larger density slope for the lower temperature.

In Fig. 4, we plot the ratio of the self-diffusion coefficient to its Enskog value, $D / D_{E}$, for several temperatures. The hard-sphere result is also plotted. The Enskog theory gives values closer to the simulation results than the Chapman-Enskog theory at all densities. Figure 4 shows the role of the correlated (and multiple) collisions for the Lennard-Jones potential. The agreement becomes better as the temperature becomes larger. This means that the binary collisions are predominant at the higher temperatures. In the hard-sphere fluid, one sees an increase of $D / D_{E}$ in the intermediate density followed by the rapid decrease in the high density region. This behavior can be semiquantitatively described by the formula proposed based on the mode-coupling theory; ; $^{815,24,29}$ 


$$
D=\frac{D_{E}}{1+R_{\rho}}+R_{t},
$$

where $R_{\rho}$ and $R_{t}$ are the contributions from the coupling of the solute motion to the density and the transverse current fluctuations, respectively. In their various original expressions, there are minor differences and extra contributions from other collective modes but they are not essential. In the low density limit, both $R_{\rho}$ and $R_{t}$ are negligible but as the density increases, the transverse current term $R_{t}$ starts to increase first to give a positive contribution to diffusion. It is due to the backflow effect where the long-time and longranged transverse current fluctuations make a vortex-shaped flow around the atom which enhances diffusion. The decrease at even higher density is attributed to the rapid increase of the density mode contribution, $R_{\rho}$. The increase in $R_{\rho}$ indicates the emergence of cage effect on diffusion.

For the Lennard-Jones fluid, however, we do not observe any increase in $D / D_{E}$ in the intermediate density. Instead it decreases monotonically as the density increases. The decrease in the high density region can be explained by the same "caging" scenario as for the hard-sphere fluid. On the other hand, the absence of the increase in $D / D_{E}$ at the intermediate density indicates the absence of any significant backflow effect, which is indeed surprising. The backflow originates from the coupling of solute's motion with the long-range hydrodynamic fluctuations. Thus, it is expected to be insensitive to the details of intermolecular potential.

There could be two obvious explanations for this sensitivity. The first scenario is that the transverse current fluctuation is indeed very sensitive to the shape of the intermolecular potential. $R_{t}$ for the Lennard-Jones fluid might be smaller than that for the hard-sphere fluid, whereas other contributions, $D_{E}$ and $R_{\rho}$, remain quantitatively similar for both potentials. The second scenario is that $R_{t}$ is not much different for both fluids but $D_{E} /\left(1+R_{\rho}\right)$ is much bigger in the Lennard-Jones than in the hard-sphere fluid. If the first scenario is true, one will observe a much smaller long-time tail in the simulation of the memory kernel of the Lennard-Jones fluid. The time window of the simulations shown in Fig. 1 is not long enough to check the presence of the long-time tails. One needs to simulate a much longer time region to choose between these two explanations.

There could be an additional scenario which is a bit more subtle. This involves multiple collisions in fluids for continuous potentials. For the hard-sphere potential, the collision is instantaneous and well defined. The possibility for three or more particles to meet at one time is very small and thus can be simply neglected. Therefore, the deviation from the Enskog values can be attributed to the correlated collisions. For the continuous potentials, however, the definition of a binary collision becomes more ambiguous as the density increases since a third particle could always be there nearby. This multiple collision effect due to three or more particles is not included in Eq. (36). We have little knowledge on how to incorporate this effect into the scheme of the mode-coupling theory and how this affects the transport coefficients. At present none of the three scenarios mentioned previously can be excluded from the argument given to explain the density dependence of the shape of the memory kernels in Sec. III.

\section{CONCLUSIONS}

In this paper, we have generalized the Enskog theory for the self-diffusion coefficient and the corresponding memory kernel to fluids with continuous potentials. Our starting point is the Green-Kubo formula based on the Mori-Zwanzig projection-operator method. The expression derived in Sec. II can be used for fluids with arbitrary potentials. Advantages of our expression over previous works are that one can relate the Green-Kubo formula to the well-known ChapmanEnskog expression in a straightforward manner in the low density limit and there is no need to solve the equation of motion for a long time in order to get the self-diffusion coefficient. We evaluated both the time-dependent memory kernel and the self-diffusion coefficient for the LennardJones fluid. Agreement of the calculated memory kernel with the simulation result is excellent at low density. As the density increases, the negative tail in the simulation becomes smaller monotonically, whereas the Enskog theory predicts exactly the opposite tendency. The self-diffusion coefficient evaluated from our theory was compared with the Chapman-Enskog theory and also with simulation value systematically for various densities and temperatures. It was found that the Enskog theory gives very good agreement for the Lennard-Jones fluid. For all the temperatures considered, the deviations are within $10 \%$ up to $\rho^{*}=0.5$. We also found that the Enskog theory is a good approximation for the higher temperature. The density dependence of $D / D_{E}$ for the Lennard-Jones fluid is entirely different from that of the hard-sphere fluid. For the Lennard-Jones fluid, there is no increase of $D / D_{E}$ in the intermediate density region which is significant (amounting to as much as 20\%) for the hardsphere fluid. Instead, it exhibits a monotonic decrease. Even at very high temperature, $T^{*}=6.0$, where one might expect much less influence of the attractive part of the potential and, therefore, the potential is effectively closer to the hard-core repulsion, we could not see any evidence of the positive backflow effect. This result might indicate that the effects of correlated and multiple collision to the transport coefficients are very sensitive to the shape of potentials.

The above-mentioned facts may change the conventional mode-coupling scenario which has been accepted as the only first-principle theory to predict the transport phenomena, even if semiquantitatively. The mode-coupling theory is based on the assumption that the dynamical processes in fluids can be separated into fast binary collisions and long correlated processes. For Lennard-Jones fluid, the concept of binary collision itself gets more ambiguous as the density increases. Third or more particles in the neighborhood might change the nature of the collision very much. Thus, one might need to include the effects of multiple collisions for continuous potentials.

In the small density region, the effect of the bounded trajectories (where the particle is trapped by the attractive part of the potential) is not very important above $T^{*}>1.0{ }^{26}$ But for larger densities, this cannot be true and the local thermal activation processes which lead to the break-up of 
the bounded trajectories might play a big role. Incorporation of such processes into the mode-coupling theory has not yet been tried.

The high frequency response of a liquid is required in the theoretical analyses of many chemical processes such as vibrational energy relaxation (VER) and barrier crossing dynamics. $^{7}$ In these cases, one often requires response at frequencies exceeding $100 \mathrm{~cm}^{-1}$. The friction at such high frequencies is expected to be dominated by the binary collisions of the type considered here. Thus, the results obtained here could be useful in the studies of VER and barrier crossing dynamics.

In this paper, we have focused only on the LennardJones fluid. The results for other continuous potentials will be considered elsewhere. ${ }^{30}$

Similar progress could be made for other transport coefficients such as the shear viscosity which should be of more practical importance because here we expect the Enskog theory is far more reliable than the Chapman-Enskog theory (judging from the hard-sphere fluid). Finally, our results should be combined with the mode-coupling type theory. The calculation in these directions is in progress. ${ }^{31}$

\section{ACKNOWLEDGMENTS}

We thank Professor Arun Yethiraj for fruitful discussion and for providing us with the data of the Monte Carlo simulation for $g(r)$ used in the present paper. We are indebted to Professor Sergei A. Egorov for drawing our attention to Ref. 10 and for discussion. We thank Professor I. M. de Schepper for encouragement and enlightening discussion. We are grateful to Dr. T. Yamaguchi for sending us the raw data for the memory kernels which were used in this paper. The work is supported in part by grants from the Japan Society for the Promotion Science (JSPS) and DST, India. G. S. thanks CSIR for a research fellowship.
${ }^{1}$ P. Résibois and M. de Leener, Classical Kinetic Theory of Fluids (Wiley, New York, 1977).

${ }^{2}$ B. J. Alder and T. E. Wainwright, Phys. Rev. A 1, 18 (1970).

${ }^{3}$ J. J. Erpenbeck and W. W. Wood, Phys. Rev. A 43, 4254 (1991).

${ }^{4}$ J. P. Boon and S. Yip, Molecular Hydrodynamics (Dover, New York, 1980).

${ }^{5}$ J. P. Hansen and I. R. McDonald, Theory of Simple Liquids, 2nd ed. (Academic, London, 1986).

${ }^{6} \mathrm{U}$. Balucani and M. Zoppi, Dynamics of the Liquid State (Oxford University Press, Oxford, 1994).

${ }^{7}$ B. Bagchi and R. Biswas, Adv. Chem. Phys. 109, 207 (1999).

${ }^{8}$ L. Sjögren and A. Sjölander, J. Phys. C 12, 4369 (1979).

${ }^{9}$ J. A. Leegwater, J. Chem. Phys. 94, 7402 (1991).

${ }^{10}$ K. N. Pathak, S. Ranganathan, and R. E. Johnson, Phys. Rev. E 50, 1135 (1994).

${ }^{11}$ R. K. Sharma, K. Tankeshwar, K. N. Pathak, S. Ranganathan, and R. E. Johnson, Phys. Rev. E 55, 1550 (1997).

${ }^{12}$ R. K. Sharma, K. Tankeshwar, and K. N. Pathak, J. Chem. Phys. 108, 2919 (1998).

${ }^{13}$ H. Mori, Prog. Theor. Phys. 33, 399 (1965); 33, 423 (1965).

${ }^{14} \mathrm{R}$. Zwanzig, in Lectures in Theoretical Physics, edited by W. E. Britten, B. W. Downs, and J. Downs (Springer, New York, 1961), p. 135.

${ }^{15}$ G. F. Mazenko and S. Yip, in Modern Theoretical Chemistry, edited by B. J. Berne (Plenum, New York, 1977), Vol. 6, p. 181.

${ }^{16}$ D. A. McQuarrie, Statistical Mechanics (Harper Collins, New York, 1973).

${ }^{17}$ J. O. Hirschfelder, C. F. Curtiss, and R. Byron Bird, Molecular Theory of Gases and Liquids (Wiley, New York, 1954).

${ }^{18} \mathrm{~S}$. Chapman and T. Cowling, The Mathematical Theory of Non-Uniform Gases (Cambridge University Press, Cambridge, 1939).

${ }^{19}$ L. D. Landau and E. M. Lifshitz, Mechanics, 3rd ed. (Pergamon, New York, 1973).

${ }^{20}$ W. G. Madden and S. A. Rice, J. Chem. Phys. 72, 4208 (1980).

${ }^{21}$ T. Yamaguchi, Y. Kimura, and N. Hirota, Mol. Phys. 94, 527 (1998).

${ }^{22} \mathrm{~T}$. Yamaguchi (private communication).

${ }^{23}$ I. M. de Schepper and E. G. D. Cohen, Phys. Lett. A 68, 308 (1978).

${ }^{24}$ R. I. Cukier and J. R. Mehaffey, Phys. Rev. A 18, 1202 (1978).

${ }^{25}$ T. R. Kirkpatrick and J. C. Nieuwoudt, Phys. Rev. A 33, 2658 (1986).

${ }^{26}$ C. F. Curtiss, J. Chem. Phys. 97, 7679 (1992).

${ }^{27}$ J. M. Kincaid, R. F. Tuo, and M. Lopez De Haro, Mol. Phys. 81, 837 (1994).

${ }^{28}$ D. M. Heyes, Phys. Rev. B 37, 5677 (1988).

${ }^{29}$ P. Résibois and J. L. Lebowitz, J. Stat. Phys. 12, 483 (1975), P. Résibois, ibid. 13, 393 (1975).

${ }^{30}$ K. Miyazaki, G. Srinivas, and B. Bagchi (unpublished).

${ }^{31}$ S. Egorov, K. Miyazaki, G. Srinivas, and B. Bagchi (unpublished). 